\title{
RAPD-PCR based genomic characterization of two populations of Culex quinquefasciatus (Diptera : Culicidae)
}

\author{
T. Kaura, P. Kaushal, M. Bansal and S. Chaudhry*
}

Mosquito Cytogenetics Unit, Department of Zoology, Panjab University, Chandigarh-160014, INDIA

* Corresponding author. E-mail: schaudhry2003@yahoo.com

\begin{abstract}
The present paper deals with the RAPD-PCR based genomic characterization of Culex quinquefasciatus Say which is a major vector of filariasis in several parts of the Indian subcontinent. One population of the test organism used in the study was procured from Goa (pop.A) while the other (pop.B) was collected from a village Nadasahib (20 kms from Chandigarh). The RAPD-PCR amplification of whole body homogenate of freshly hatched individual specimens was carried out by using three random primers: primer I- 5'- GTCCCGACGA - 3'; primer II- 5' - TGATCCCTGG - 3' and primer III- 5'- GTGACGTAGG - 3'. Primer I produced 5 distinct bands from the DNA of pop. A, whose base composition ranged from 200-1000 bp. Likewise, 7 bands ranging from 130-750 bp and 4 bands ranging from $270-950$ bp were generated with primers II and III respectively. In case of pop.B, a total of 8 bands ranging from 200-1000 bp were generated with primer I. Similarly, a total of 6 bands ranging from $250-$ $900 \mathrm{bp}$ with primer II and 4 bands ranging from 180-950 bp with primer III were produced. Based on the band sharing coefficient and the application of Nearest Neighbour Joining (NJ) analysis it was found that primer I was more suitable for detecting genomic differences at the species and generic levels while primer II was ideal for detecting variations in the number of bp in RAPD generated bands among different populations of $C x$. quinquefasciatus.
\end{abstract}

Keywords: PCR, Cx. quinquefasciatus, Genomics \section{INTRODUCTION \\ The techniques of molecular cell biology have helped in} providing valuable addition to the already existing data on the genetic properties of species derived from their chromosome features. Although, cytogenetic investigations on Culex quinquefasciatus was a starting point for genome studies on mosquitoes (Stevens, 1910) yet much of the work was carried out on species of the genus Anopheles. The main reason for this had been the presence of poor quality polytene chromosomes inCulex and lack of variation in the diploid number of 6 chromosomes $(2 n=6)$ which has a remarkable constancy in the family Culicidae. In mosquitoes, the phenomenon of sibling species has provided enough impetus to carry out molecular level genome characterization to detect species and their subspecific variants (Beebe and Saul, 1995; Cornel et al., 1996; Rutledge et al., 1999; Chaudhry, 1999; Beebe et al., 2000; UC Davis: News Information, 2002; Bhargavi et al., 2005; and Lanzaro et al., 2009). In order to manipulate DNA in the in vitro condition, the technique of Polymerase Chain Reaction (PCR) has actually revolutionized the studies aimed at DNA diagnostics of cryptic species (Wilkerson et al., 1993, 1995; Hill and Crampton, 1994; and Collins and Paskewitz, 1996). Since the introduction of PCR based genomic analysis, bulk of the information relates to species of genera Anopheles and Aedes due to their role as potential vectors of malaria, yellow fever, dengue and dengue haemorhagic fever (Ballinger-Crabtree et al., 1992; Beebe et al., 2000; 1992; and Urdaneta et al., 2005). Comparatively less is known about the species of the genus Culex which also include species of epidemiological significance. For the PCR based enzymatic amplification of mosquito genome, random as well as gene specific primers has been developed for selected nuclear and mitochondrial gene sequences (Severini et al., 1996; Crabtree et al., 1995; Cornel et al. 1996; Proftet al., 1999; Manonmani et al., 2001; Singh et al., 2004; and Li and Wilkerson, 2005). In fact, the major impetus for mosquito genome studies came from WHO/UNDP/World Bank (2003) special programme for research and training in tropical diseases. In this document recommendations were made for $C x$. quinquefascaiatus genome project on the pattern of Anopheles gambiae genome project (Holt et al., 2002). In reference to these developments in the subject area of mosquito cytogenetics, the present research work was aimed at the RAPD-PCR based genomic analysis of two allopatric populations of Culex quinquefascaiatus Say so as to find out a suitable DNA marker for the identification of various populations of this taxon.

\section{MATERIALS AND METHODS}

Culex quinquefasciatus is a common house mosquito in 
the entire Oriental region including India where it is responsible for the transmission of filariasis and a number of viral infections in human beings. For the present purpose of research, one population was procured from Goa (pop.A) while the other (pop.B) was collected from a village Nadasahib (20 kms from Chandigarh). The RAPDPCR amplification was carried out as per the standard protocols of Sambrook etal. (1989), Wilkersonetal. (1993) and Ausubel et al. (1999). The three ten base oligonucleotide primers used for the random amplification of complementary segments of DNA were: primer I- 5'- GTCCCGACGA - 3'; primer II- 5' TGATCCCTGG - 3' and primer III- 5'- GTGACGTAGG 3'. The statistical analysis of PCR amplified bands generated with these primers was carried out for constructing the dendrograms of phylogenetic kinship which was based on accurate pictures of unique and shared DNA bands produced from the two allopatric populations of the species.

DNA extraction and amplification: For the extraction of DNA, three freshly hatched unfed specimens of each population were used in which one specimen at a time was processed according to the phenol-chloroform extraction method of Ausubel et al.(1999). Accordingly, the whole body homogenate was prepared in 50-100 $\mu \mathrm{l}$ lysis buffer consisting of $10 \mathrm{mM}$ Tris- $\mathrm{HCl}, 1 \mathrm{mM}$ EDTA, $25 \mathrm{Mm} \mathrm{NaCl}$ and $1 \%$ SDS for the lysis of cell membranes. These contents were incubated at $37^{\circ} \mathrm{C}$ for $30 \mathrm{mins}$ in a water bath after which $5 \mu$ l Proteinase K $(20 \mathrm{mg} / \mathrm{ml})$ was added and the contents were incubated at $65^{\circ} \mathrm{C}$ for $1 \mathrm{hr}$ in a water bath. While the tubes were still warm, $100 \mu 13 \mathrm{M}$ sodium acetate was also added and again incubated on ice for $1 \mathrm{hr}$. These contents were centrifuged at $4^{\circ} \mathrm{C}$ for 10 mins at 10,000 rpm. The resulting supernatant was transferred to fresh eppendorf tube to which $100 \mu$ ls each of phenol and chloroform+ isoamyl alcohol were added. These tubes were again centrifuged at $8000 \mathrm{rpm}$ for 10 mins at $4{ }^{\circ} \mathrm{C}$ after which upper aqueous layer containing the DNA was transferred to fresh eppendorf tube to which twice the volume of chilled $\left(-20^{\circ} \mathrm{C}\right)$ ethanol was added before storing them overnight at $-20^{\circ} \mathrm{C}$. These tubes were again centrifuged for $10 \mathrm{~min}$. after which the aliquot was discarded without disturbing the DNA pellet. The pellet were washed gently with $70 \%$ ethanol and dried after which it was also dissolved in $20 \mu \mathrm{l}$ of Tris EDTA (TE) buffer for maintaining the $\mathrm{pH}$ before storage at $4^{\circ} \mathrm{C}$. The purity of extracted sample of DNA was determined by spectrophotometric analysis in which its concentration ranged between 1.7-2.0ng/ $\mu \mathrm{l}$ from different samples.

The PCR thermocycler was programmed for the following temperature variables as: 1 cycle for initial denaturation of DNA at $94^{\circ} \mathrm{C}$ for 10 mins followed by a cycle of denaturation, annealing of primer and extension of DNA at $94^{\circ} \mathrm{C}$ for $1 \mathrm{~min}, 37^{\circ} \mathrm{C}$ for $1 \mathrm{~min}$ and $72^{\circ} \mathrm{C}$ for 1 min respectively. This cycle of 3 mins was repeated a total of 45 times followed by one cycle of final extension at $72^{\circ} \mathrm{C}$ for $10 \mathrm{~min}$. In all such amplification cycles, a negative control consisting of all the components of reaction mixture except the DNA, was also loaded so as to rule out the experimental errors. The PCR end products were then subjected to $2 \%$ agarose gel electrophoresis containing ethidium bromide (EtBr) as a dye with a concentration of $0.5 \mathrm{mg} / \mathrm{ml}$ using $1 \mathrm{X}$ TAE buffer at a constant voltage of 50 volts. The DNA bands which appeared in the gel were observed and photographed immediately over UV transilluminator. In one of the lanes, a 100 to 1000 base pair standard DNA ladder was also run along with all the PCR products for calculating the number of base pairs in each band of DNA generated through this process. The base pair number of all the amplified DNA fragments was calculated by using a standard curve. For this the electrophoretic mobility of each fragment was calculated by using the following formula (Sambrook et al., 1989):

\section{$\begin{aligned} \text { Relative mobility } & \text { Distance traveled by the DNA } \\ & \text { Distance traveled by the dye BPB (Bromophenol blue) }\end{aligned}$}

Depending upon the presence or absence of DNA bands generated from the individuals of the two populations of the species, the data was scored as 1 if the band was present and 0 when absent. From these values the band sharing coefficient between individuals of the two populations (individuals marked as a and $\mathrm{b}$ respectively) was calculated by employing the formula: $\mathrm{D}=2 \mathrm{~N}_{\mathrm{ab}}$ / $\left(\mathrm{N}_{\mathrm{a}}+\mathrm{N}_{\mathrm{b}}\right)$ where D denotes band sharing coefficient, $\mathrm{N}_{\mathrm{ab}}-$ number of bands shared by individuals a and $\mathrm{b}, \mathrm{Na}$ number of bands obtained from a and $\mathrm{N}_{\mathrm{b}}$-number of bands obtained from $b$. It is based on the principle that the increase in the value of band sharing coefficient

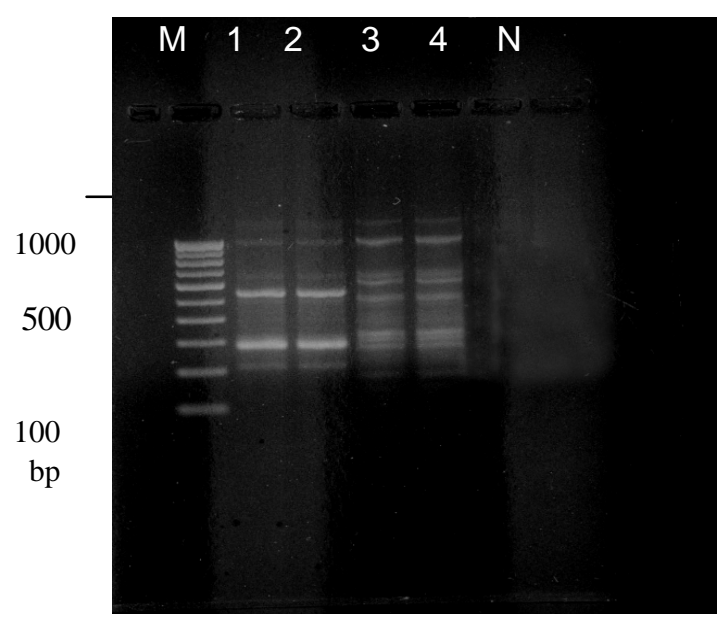

Fig.1. DNA bands generated with primer I. Lane M-gene ruler, lane $N$ : negative control, 1: ( pop.A), 2: (pop. A), 3: (pop.B), 4: (pop.B) of Cx. quinquefasciatus. 


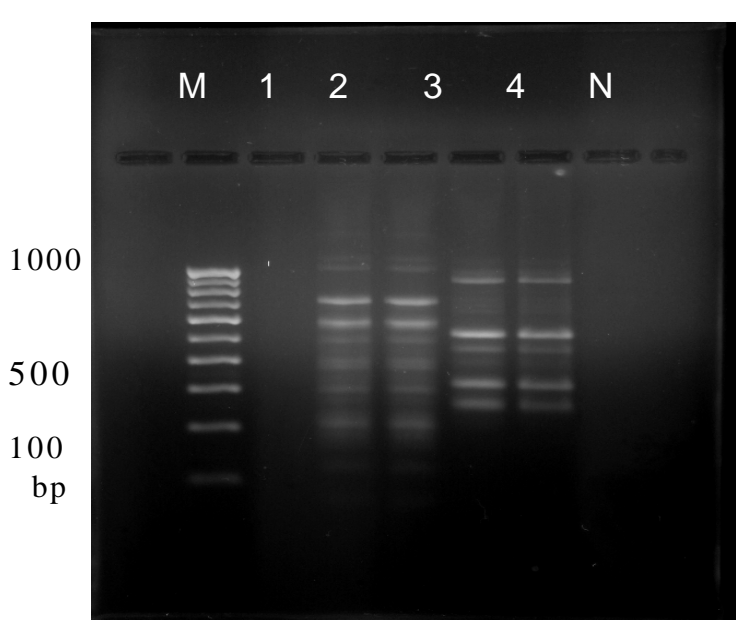

Fig.2. DNA bands generated with primer II. Lane M-gene ruler, lane $N$ : negative control, 1: (pop.A), 2: (pop.A), 3: (pop.B), 4: (pop.B) of Cx. quinquefasciatus.

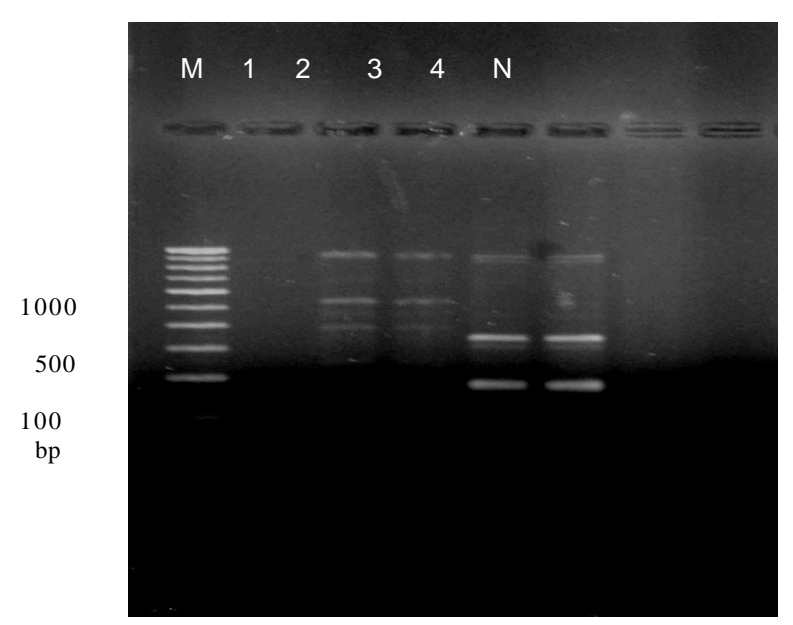

Fig.3. DNA bands generated with primer III. Lane M-gene ruler, lane $N$ - negative control, 1: (pop.A), 2: (pop.A), 3: (pop.B), 4: (pop.B), of Cx. quinquefasciatus.

\section{With primer I:}

Rescaled Distance Cluster Combine

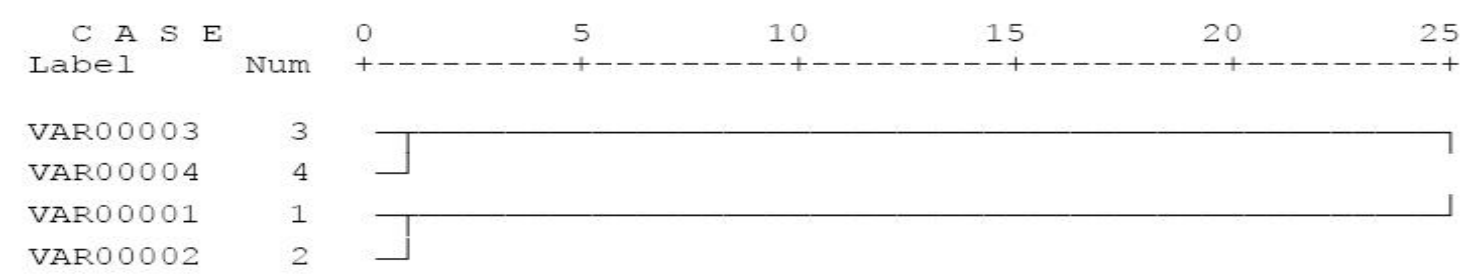

\section{With primer II:}

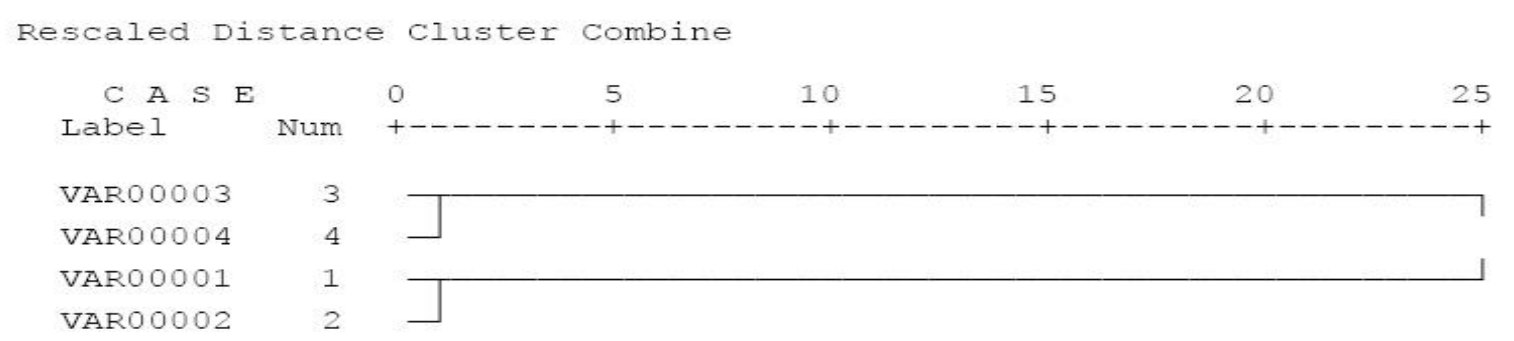

\section{With primer III:}

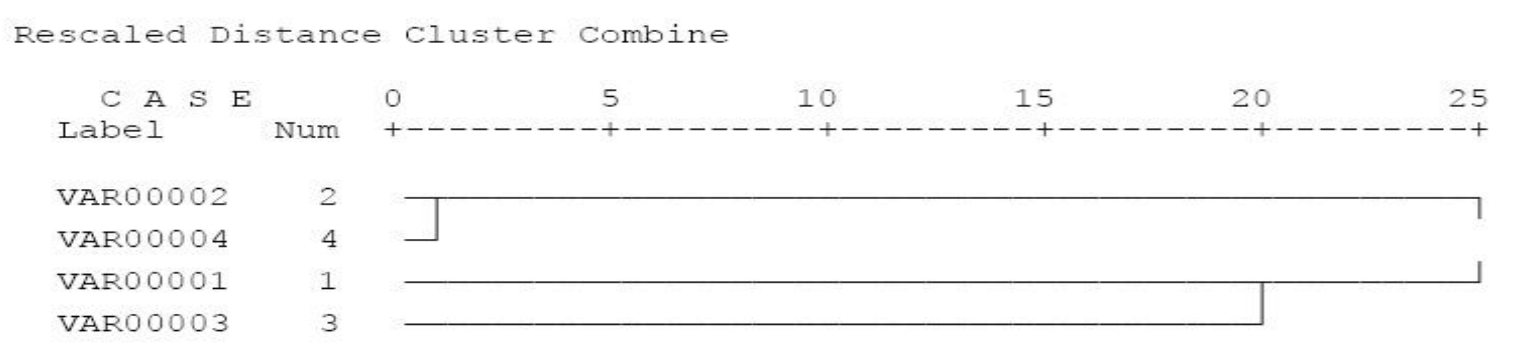

Fig.4. Dendrograms showing genomic relatedness between two populations (pop.A, pop.B) of Cx. quinquefasciatus. 1, 2Individuals of pop.A, 3, 4-Individuals of pop.B. 
Table 1. Presence absence (+,-) data of all the DNA bands produced with primers I, II and III from populations A and B of $C x$. quinquefasciatus.

\begin{tabular}{|c|c|c|c|c|}
\hline \multirow{2}{*}{$\begin{array}{l}\text { Primer based Appx. bp } \\
\text { number in the amplified } \\
\text { DNA fragment } \\
\end{array}$} & \multicolumn{4}{|c|}{ Population } \\
\hline & $\overline{\text { pop.A }}$ & pop.A & pop.B & pop.B \\
\hline \multicolumn{5}{|l|}{ With primer - I } \\
\hline 1000 & + & + & + & + \\
\hline 670 & + & + & + & + \\
\hline 610 & - & - & + & + \\
\hline 550 & + & + & - & - \\
\hline 520 & - & - & + & + \\
\hline 350 & - & - & + & + \\
\hline 300 & + & + & + & + \\
\hline 290 & - & - & + & + \\
\hline 220 & + & + & - & - \\
\hline 200 & - & - & + & + \\
\hline \multicolumn{5}{|l|}{ With primer - II } \\
\hline 900 & - & - & + & + \\
\hline 750 & + & + & - & - \\
\hline 580 & + & + & - & - \\
\hline 490 & - & - & + & + \\
\hline 470 & + & + & - & - \\
\hline 420 & - & - & + & + \\
\hline 380 & + & + & + & + \\
\hline 320 & - & - & + & + \\
\hline 300 & + & + & - & - \\
\hline 250 & - & - & + & + \\
\hline 220 & + & + & - & - \\
\hline 130 & + & + & - & - \\
\hline \multicolumn{5}{|l|}{ With primer - III } \\
\hline 950 & + & + & + & + \\
\hline 900 & - & - & + & + \\
\hline 570 & + & + & - & - \\
\hline 400 & + & + & - & - \\
\hline 330 & - & - & + & + \\
\hline 320 & - & - & + & + \\
\hline 270 & + & + & - & - \\
\hline 180 & - & - & + & + \\
\hline $\begin{array}{l}\text { increases the inter and/ or } \\
\text { relatedness among the member } \\
\text { populations of the same specie } \\
\text { RESULTS AND DISCUS } \\
\text { The amplification with prim } \\
\text { bands from the DNA of pop. A } \\
\text { ranged from } 200-1000 \mathrm{bp} \text {. Li } \\
\text { from } 130-750 \mathrm{bp} \text { and } 4 \text { bands } ~ \\
\text { were generated with primers II }\end{array}$ & $\begin{array}{l}\text { intra-specific genetic } \\
\text { of different species and } \\
\text { s. } \\
\text { ION } \\
\text { I I produced } 5 \text { distinct } \\
\text { whose base composition } \\
\text { ewise } 7 \text { bands ranging } \\
\text { anging from } 270-950 \mathrm{bp} \\
\text { and III, respectively. In }\end{array}$ & \multicolumn{3}{|c|}{$\begin{array}{l}\text { case of pop.B a total of } 8 \text { bands ranging from } 200-1000 \\
\text { bp were generated with primer I. Similarly, a total of } 6 \\
\text { bands ranging from } 250-900 \mathrm{bp} \text { with primer II and } 4 \\
\text { bands ranging from } 180-950 \mathrm{bp} \text { with primer III were } \\
\text { produced respectively (Figs. } 1,2 \text { and } 3 \text { ). Since more } \\
\text { number of unique bands were obtained by using } \\
\text { primer II, therefore it was considered to be an ideal } \\
\text { marker for distinguishing the two populations of this } \\
\text { species. In addition to this, an attempt was also made } \\
\text { to construct dendrograms of phylogenetic relatedness }\end{array}$} \\
\hline
\end{tabular}


Table 2. Band sharing coefficient between populations A and B of Cx. quinquefasciatus (+ band present, - band absent).

\begin{tabular}{|c|c|c|}
\hline S.No. & Individuals analyzed & Band sharing coefficient \\
\hline \multicolumn{3}{|c|}{ With primer-I } \\
\hline 1 & Ind. 1 and 2 (pop. A) & 1 \\
\hline 2 & Ind.1 (pop.A) and Ind.3 (pop.B) & 0.46 \\
\hline 3 & Ind.1 (pop.A) and Ind.4 (pop.B) & 0.46 \\
\hline 4 & Ind.2 (pop.A) and Ind.3 (pop.B) & 0.46 \\
\hline 5 & Ind.2 (pop.A) and Ind.4 (pop.B) & 0.46 \\
\hline 6 & Ind. 3 and 4 (pop. A) & 1 \\
\hline \multicolumn{3}{|c|}{ With primer-II } \\
\hline 1 & Ind. 1 and 2 (pop. A) & 1 \\
\hline 2 & Ind.1 (pop.A) and Ind.3 (pop.B) & 0.15 \\
\hline 3 & Ind.1 (pop.A) and Ind.4 (pop.B) & 0.15 \\
\hline 4 & Ind.2 (pop.A) and Ind.3 (pop.B) & 0.15 \\
\hline 5 & Ind.2 (pop.A) and Ind.4 (pop.B) & 0.15 \\
\hline 6 & Ind. 3 and 4 (pop. A) & 1 \\
\hline \multicolumn{3}{|c|}{ With primer-III } \\
\hline 1 & Ind. 1 and 2 (pop. A) & 1 \\
\hline 2 & Ind.1 (pop.A) and Ind.3 (pop.B) & 0.22 \\
\hline 3 & Ind.1 (pop.A) and Ind.4 (pop.B) & 0.22 \\
\hline 4 & Ind.2 (pop.A) and Ind.3 (pop.B) & 0.22 \\
\hline 5 & Ind.2 (pop.A) and Ind.4 (pop.B) & 0.22 \\
\hline 6 & Ind.3 and 4 (pop. A) & 1 \\
\hline
\end{tabular}

between these two allopatric populations of $C x$. quinquefasciatus. It was observed that, with all the three primers, the individuals marked as number 1 and 2 of pop.A and individuals 3 and 4 of pop.B clustered together while they were separated by rescaled cluster combine value of 25 (Fig. 4). The production of these DNA profiles were also subjected to "Nearest Neighbour Joining (NJ) analysis" (single linkage method) of the closely placed bands (Table 1). The difference between the number of unique bands in both the populations with all the three primers were examined and primer II generated more number of unique bands than other two primers therefore, it could be considered as an ideal sequence for population level studies. In the present studies, the nearest neighbour analysis was useful in selecting a specific group of amplified fragments which could be used to differentiate the two populations. In addition to the use of these parameters of genome analysis, the band sharing analysis was also employed to find out the genetic differences among the individuals at the population level (Table 2). The value of band sharing coefficient is 1 only with both the individuals of respective populations in accordance with their allopatric status. By following the hierarchial clustand

band sharing coefficient analysis, it was seen that once again primer II was more suitable for the genomic differentiation of the populations of this species. From the present data set it was logical to conclude that primer II was more suitable for detecting variations in the number of bp among different populations of $C x$. quinquefasciatus while primer I was more suitable for detecting the differences at the species and generic levels. In the light of $C x$. quinquefasciatus Genome Project (WHO/UNDP/World Bank 2003) the present studies are considered as a significant beginning for estimating the feasibility of Culex as an ideal experimental representative for other nonanopheline taxa of the family Culicidae.

\section{ACKNOWLEDGEMENTS}

The authors are thankful to the University Grants Commission, New Delhi for providing the necessary facilities under its Special Assistance Programme (SAP) 
to the Department of Zoology, Panjab University Chandigarh.

\section{REFERENCES}

Ausubel, F.M., Breut, R., Kingston, R.E., Moore, D.D., Sideman, J.G., Smith, J.A. and Struhl, K. (1999). Short protocols in molecular biology, London: John-Wiley and Sons Inc.

Ballinger-Crabtree, M.E., Black, W.C. $4^{\text {h }}$ and Miller, B.R. (1992). Use of genetic polymorphisms detected by the random-amplified polymorphic DNA polymerase chain reaction (RAPD-PCR) for differentiation and identification of Aedes aegypti subspecies and populations. The American Journal of Tropical Medicine and Hygiene, 47: 893-901.

Beebe, N.W. and Saul, A. (1995). Discrimination of all the members of the Anopheles punctulatus complex by polymerase chain reaction - restriction fragment length polymorphism analysis. The American Journal of Tropical Medicine and Hygiene, 53: 478-481.

Beebe, N.W., Cooper, R.D., Foley, D.H. and Ellis, J.H. (2000). Populations of the south-west Pacific malaria vector Anopheles farauti s.s. revealed by ribosomal DNA transcribed spacer polymorphisms. Heredity, 84: 244- 253.

Bhargavi, R., Vishwakarma, S. and Murty, U.S. (2005). A secondary structural common core in the ribosomal ITS2 (internal transcribed spacer) of Culex species from diverse geographical locations. Bioinformation, 1(2): 52-55.

Chaudhry, S. (1999). A review of polytene chromosome dynamics in genus Anopheles (Diptera: Culicidae). In: R.C. Sobti and J.S. Yadav (Eds.) Some aspects on the insight of insect biology (pp 205-236), New Delhi: Tausco Book Distributors.

Collins, F.H. and Paskewitz, S.M. (1996). A review of the use of ribosomal DNA (rDNA) to differentiate among cryptic Anopheles species. Insect Molecular Biology, 5(1): 1-9.

Cornel, A.J., Porter, C.H. and Collins, F.H. (1996). Polymerase chain reaction species diagnostic assay for Anopheles quadrimacualtus cryptic species (Diptera: Culicidae) based on ribosomal DNA ITS2 sequences. Journal of Medical Entomology, 33(1): 109- 116.

Crabtree, M.B., Savage, H.M. and Miller, B.R. (1995). Development of a species diagnostic PCR assay for the identification of Culex vectors of St. Louis encephalitis virus based on interspecies sequence variation in ribosomal DNA spacers. The American Journal of Tropical Medicine and Hygiene, 53(1): 105-109.

Hill, S.M. and Crampton, J.M. (1994). DNA based methods for the identification of insect vectors. Annals of Tropical Medicine and Hygiene, 88: 227-250.

Holt, R.A., Subramanian, G.M., Halpern, A., Sutton, G.G., Charlab, R., Nusskern, D.R. (2002). The genome sequence of the malaria mosquito Anopheles gambiae. Science, 298(55951): 129-149.

Lanzaro, G.C., Nuzhdin, S. and Tripet, F. (2009). Tools for monitoring the genetic structure and stability of mosquito populations. pp 157-164. Retrieved April 20, 2009 from: gelanzaro@ucdavis.edu.

Li, C. and Wilkerson, R.C. (2005). Identification of Anopheles (Nyssorhynchus) albitarsis complex species (Diptera: Culicidae) using rDNA ITS2-based polymerase chain reaction primers. Memorias do Instituto Oswaldo Cruz, 100(5): 495-500.

Manonmani, A.M., Townson, H., Adeniran, T., Jambulingam, P., Sahu, S. and Vijayakumar, T. (2001). rDNA-ITS2 polymerase chain reaction assay for the sibling species of Anopheles fluviatilis. Acta Tropica, 78(1): 3-9.

Proft, J., Maier, W.A. and Kampen, H. (1999). Identification of six sibling species of the Anopheles maculipennis complex (Diptera: Culicidae) by a polymerase chain reaction assay. Parasitology Research, 85(10): 837-843.

Rutledge, C.R., Wesson, D.M. and Meek, C.L. (1999). Polymerase chain reaction assay to identify all immature stages of two species of the Anopheles quadrimaculatus sibling species complex (Diptera: Culicidae). Journal of the American Mosquito Control Association, 15(4): 573575.

Sambrook, J., Fritsch, E.F. and Maniatis, T. (1989). Molecular cloning: a laboratory manual, $2^{\text {nd }}$ ed. New York: Cold Spring Harbor.

Severini, C., Silverstrini, F., Mancini, P., La Rosa, G. and Marinucci, M. (1996). Sequence and secondary structure of the rDNA second internal transcribed spacer in the sibling species Culex pipiens L. and Cx. quiquefasciatus Say (Diptera: Culicidae). Insect Molecular Biology, 5(3): 181186.

Singh, O.P., Chandra, D., Nanda, N., Raghavendra, K., Sunil, S., Sharma, S.K., Dua, V.K. and Subbarao, S.K. (2004). Differentiation of members of the Anopheles fluviatilis species complex by an allele-specific polymerase chain reaction based on $28 \mathrm{~S}$ ribosomal DNA sequences. The American Journal of Tropical Medicine and Hygiene, 70(1): 27-32.

Stevens, N.M. (1910). The chromosomes in the germ cells of Culex. Journal of Experimental Zoology, 8: 207-217.

UC Davis News Information (2002). Mosquito Ecology Needs Study of Genetically Modified Mosquitoes are to Curb Disease. pp 1-2. Retrieved May 22, 2009. http:// www.news.ucdavis.edu/search/news_detail.

Urdaneta, L., Herrera, F., Pernalete, M., Zoghbi, N., RubioPalis, Y., Barrios, José Rivero, R., Comach, G., Jiménez, M. and Salcedo, M. (2005). Detection of dengue viruses in field-caught Aedes aegypti (Diptera: Culicidae) in Maracay, Aragua state, Venezuela by type-specific polymerase chain reaction. Infection Genetics and Evolution, 5(2): 177-184.

Wilkerson, R.C., Parsons, T.J., Albright, D.G., Klein, T.A. and Braun, M.J. (1993). Random amplified polymorphic DNA (RAPD) markers readily distinguish cryptic mosquito species (Diptera: Culicidae: Anopheles). Insect Molecular Biology, (4): 205-211.

Wilkerson, R.C., Parsons, T.J., Klein, T.A., Gaffigan, T.V., Bergo, E. and Consolim, J. (1995). Diagnosis by random amplified polymorphic DNA polymerase chain reaction of four cryptic species related to Anopheles (Nyssorhynchus) albitarsis (Diptera: Culicidae) from Paraguay, Argentina, and Brazil. Journal of Medical Entomology, 32(5): 697-704.

WHO/UNDP/World Bank, (2003). Special Programme for Research and Training in Tropical Diseases (TDR). Molecular Entomology. 1-5. 